\title{
Black Mold: A Case Presentation and Discussion of Cutaneous Stachybotrys chartarum Infection
}

\author{
Noura Ayoubi ${ }^{1 *}$ and Vadin Lall Dass ${ }^{2}$ \\ ${ }^{1}$ Morsani College of Medicine, University of South Florida, USA \\ ${ }^{2}$ Department of Internal Medicine, James A. Hayley Veterans Association, USA
}

\begin{abstract}
Stachybotrys chartarum, also known as black mold, is a type of indoor fungus that grows in areas of high moisture and humidity. At high rates of growth, the fungus can produce mycotoxins that become airborne and inhaled. More often than not, inhalation of significant amounts of black mold causes pulmonary mycotoxicosis. Here we present a case of a cutaneous black mold infection of the scalp in an 80-year-old male with no history of pulmonary mycotoxicosis with Stachybotrys chartarum. This case is presented because it illustrates the rare incidence of Stachybotrys chartarum in the context of a cutaneous infection rather than pulmonary mycotoxicosis. This type of fungus is difficult to culture and takes time to grow. It is important for physicians to be aware of its potential occurrence in patients with cutaneous infections because treatment is much more rigorous than that of simple bacterial skin infections. Recognition of this fungal growth early on can help patients get treated with the necessary medications before extensive spread of the mold can occur.
\end{abstract}

\section{Keywords}

Black mold, Stachybotrys chartarum

\section{Introduction}

Stachybotrys chartarum, more commonly known as black mold, is a type of indoor fungus that grows in areas of high moisture and humidity [1]. It is also known as Stachybotrys atra and Stachybotrys alternans. The striking greenblack color is a result of dark melanin pigment produced by the fungus. Figure 1 illustrates the appearance of the mold in the environment [2].

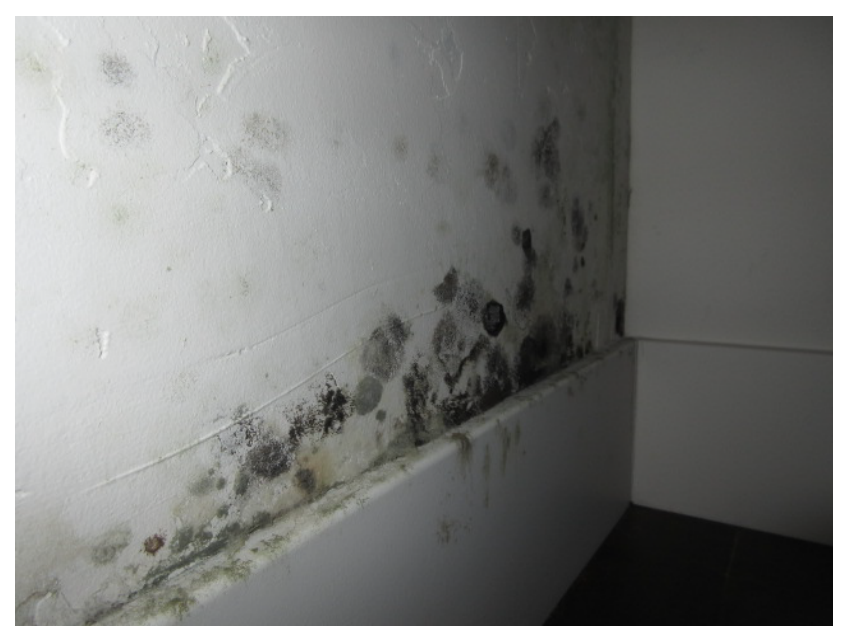

Figure 1: Stachybotrys chartarum growth on the wall of an indoor building (Centers for Disease Control and Prevention).
Black mold can be found in soil and grains but is most common in damp buildings [3]. It has a high affinity for cellulose-rich environments, which explains why this fungus is difficult to culture on standard media. Additionally, it takes longer to grow than more common fungi, such as Aspergillus spp. or Rhizopus spp [4]. Unless a culture media is cellulose-rich, $S$. chartarum is often taken over by other types of fungi. Figure 2 shows a culture of Stachybotrys that was obtained from a contaminated wall [5].

At high rates of growth, the fungus can produce mycotoxins that become airborne and inhaled. Inhalation of significant amounts of black mold causes pulmonary mycotoxicosis. Patients present with flu-like symptoms that include fever, chills, nausea, cough, dyspnea, headache and myalgia [5]. Infections are usually self-limited, requiring only supportive care [5]. Here we present a case of cutaneous S. chartarum infection during which the patient did not develop any

\begin{abstract}
*Corresponding author: Noura Ayoubi, BS, Morsani College of Medicine, University of South Florida, 12901 Bruce B. Downs Blvd, Tampa, FL 33612, USA, Tel: (813)-455-9606
\end{abstract}

Accepted: August 28, 2019

Published online: August 30, 2019

Citation: Ayoubi N, Dass VL (2018) Black Mold: A Case Presentation and Discussion of Cutaneous Stachybotrys chartarum Infection. Dermatol Arch 3(1):80-81 


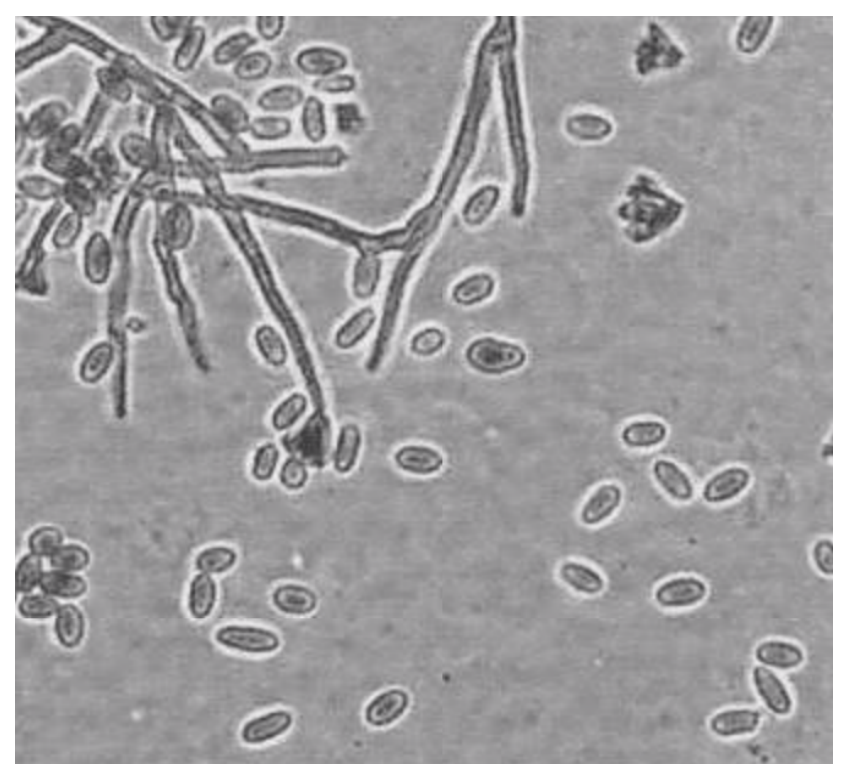

Figure 2: Culture of Stachybotrys at 40x [5].

pulmonary symptoms. This case report highlights the rarity of cutaneous black mold infection and delves into methods of diagnosis and standards of treatment.

\section{Case Presentation}

Here we present a case of a cutaneous black mold infection of the scalp in an 80-year-old immunocompetent male with no history of pulmonary mycotoxicosis with Stachybotrys chartarum. The patient presented with a subcutaneous nodule on his temporal scalp area that had been growing for the past few weeks. At the time, differential diagnoses included lipoma, abscess or malignancy. Imaging of the head revealed extensive skin involvement with potential extension to the dura so emergent surgery was performed to remove the mass and determine a histopathological diagnosis. The patient was treated with antibiotics due to a concern of Staphylococcus aureus infection. However, after a 10day culture of the excised area, black mold began to grow on standard culture media. The patient was brought back in for further debridement involving wider margins in order to ensure no more fungus was left behind. He was placed on a year-long treatment regimen consisting of amphotericin $B$ and was discharged for follow-up in outpatient clinic.

\section{Discussion}

This case is presented because it illustrates the rare incidence of Stachybotrys chartarum in the context of a cutaneous infection rather than pulmonary mycotoxicosis. In our case, the differential diagnoses list included Staphylococcus aureus infection. Continuing to treat the patient with antibiotics would not have resulted in resolution of symptoms. Rather, the infection could have extended into the dura, warranting much more extensive treatment.

This type of fungus is difficult to culture and takes time to grow. A more evolving approach for diagnosis of fungal infections is DNA sequencing. This can be utilized to make a diagnosis more quickly when there is a high index of clinical suspicion. However, this can be difficult because other fungi also produce dark melanin pigment but do not produce mycotoxins. The term black mold simply refers to the black appearance of the mold as it grows in the environment.

The most common indoor fungi include Alternaria, Aspergillosis, Cladosporium, and Penicillum [6]. The incidence of Stachybotrys is far less common. The mold preferentially grows in cellulose-rich environments with low nitrogen. This type of environment can be found surrounding material such as fiberboard, dust, paper, and lint [6]. Additionally, the mold requires moisture for growth so water damage to any of the aforementioned material will provide the perfect environment for growth of Stachybotrys.

In addition to treating fungal infections, it is also important to counsel patients on management of the fungus source so that they are not re-infected. First and foremost, the Centers for Disease Control and Prevention (CDC) recommends that patient clean the moldy surface with commercial products or a water-bleach solution [6]. In cases of extensive mold growth, professionals should be contacted. In order to prevent regrowth, the CDC highlights keeping humidity low by using air conditioners or dehumidifiers [6]. Additionally, mold-inhibitors or mold-killers can also be used [6].

It is important for physicians to be aware of potential Stachybotrys etiology in patients with cutaneous infections because treatment is much more rigorous than that of simple bacterial skin infections. Nevertheless, S. chartarum infection is still treatable with a prolonged course amphotericin B. Recognition of this fungal growth early on can help patients get treated with the necessary medications before extensive spread of the mold can occur.

\section{Conflicts of Interest}

The authors have no conflicts of interest to declare.

\section{References}

1. Johanning E, Biagini R, Hull D, et al. (1996) Health and immunology study following exposure to toxigenic fungi (Stachybotrys chartarum) in a water-damaged office environment. Int Arch Occup Environ Health 68: 207-218.

2. (2015) Sierck, P. Stachybotryschartarum (atra). ET\&T: Indoor Environmental Surveys.

3. Samson RA, Houbraken J, Thrane U, et al. (2010) Food and Indoor Fungi. CBS-KNAW Fungal Biodiversity Centre. Utrecht, the Netherlands, 1-398.

4. Pestka JJ, Yike I, Dearborn DG, et al. (2008) Stachybotrys chartarum, trichothecene mycotoxins, and damp buildingrelated illness: new insights into a public health enigma. Toxicol Sci 104: 4-26.

5. Kuhn DM, Ghannoum MA (2003) Indoor mold, toxigenic fungi, and Stachybotrys chartarum: Infectious disease perspective. Clin Microbiol Rev 16: 144-172.

6. https://www.cdc.gov/mold/stachy.htm 